prevalence was the highest in young people, with $3.6 \%$ for women and $2.4 \%$ in men aged $18-24$ yo. To face this problem, several countries have developed new strategies, mixing newer technologies and home-based self-sampling test. Inspired by evaluation of those dispositives, the French National Institute of Health Prevention and Education (INPES) decided to experiment the proposition of a free home-based self-sampling to screen this infection via internet in young people 18-24 yo. This study, named Chlamyweb, aims to compare CT screening rate from this intervention with traditional information system and screening centre. Study design was a random control trial, with a 1:2 randomization. Recruitment took place on an Internet information website on sexually transmitted infections, and support by web campaign from September 3 to October 14 2012. Home-based kits were composed by uriswab 3 sponges for men and dry vaginal swabs for women (Copan diagnostics). All samples were analysed by using the fully automated cobas 4800 (Roche diagnostics). Self-sampling was proposed to 5531 people. Out of them, $47.3 \%$ accepted, with a higher rate in women (53.0\%). A total of 1616 kits provided [1002 from women (63.8\%) and 614 from men (58.8\%)] was return to the French National Reference Center for chlamydial infections. The global prevalence was $6.8 \%$ (8.3\% in women, $4.4 \%$ in men). Sexual behaviour and sociodemographic patient's characteristics were collected and their analysis is under investigation. These preliminary results show that Internet testing reaches a population with a high prevalence of CT infection and appears to be acceptable to young people.

\section{P3.026 LOW PREVALENCE OF CHLAMYDIA TRACHOMATIS INFECTION: FACT OR MISSED DIAGNOSIS?}

doi:10.1136/sextrans-2013-051184.0486

T Bharara, D Rawat, P Bhalla, V K Garg, K Sardana, V Patwardhan. Maulana Azad Medical College, New Delhi, India

Background Chlamydia trachomatis is reported to be the most common sexually transmitted infection (STI) in developed countries; this data depends on the sensitivity and specificity of the diagnostic test employed. Even when Nucleic acid amplification tests (NAATs) are used to detect C.trachomatis infection, the occurrence of variant strains which lack the cryptic plasmid or possess deletion mutations may be responsible for negative test results, as was detected in Sweden recently. The present study was undertaken to establish the true prevalence of C.trachomatis infection among males with urethritis using not only a well tested NAAT but also Direct Fluorescent Antibody test (DFA).

Methods A total of 34 male patients reporting to the STI OPD of the Lok Nayak Hospital with urethritis were included in this study. Gram staining and culture was done for detection of Neisseria gonorrhoeae and DFA and real-time PCR using COBAS ${ }^{\circledR}$ TaqMan $^{\circledR}$ CT Test, v2.0 to detect the presence of C.trachomatis.

Results Among the 34 males, $55.9 \%$ cases were positive for N.gonorrhoeae by culture. DFA for C.trachomatis was positive in $17.6 \%$ cases, 5 out of 6 DFA positive cases were also positive for N.gonorrhoeae. PCR for C.trachomatis has so far been done in 19 cases, including 4 of the DFA positive cases, and none of them yielded a positive result. Neither C.trachomatis nor N.gonorrhoeae was found in $41.1 \%$ cases.

Conclusion C.trachomatis was detected only in $17.6 \%$ men with urethritis and that too only by DFA and none by PCR. This could be either an actual low prevalence of C.trachomatis or it may be due to the occurrence of new variant strains not detected by the molecular diagnostic test used by us. Sequencing of C.trachomatis isolated from patient specimens will be required to detect mutants that could be responsible for underdiagnosis, when relying only on NAATs.

\section{P3.027 PREVALENCE AND DETERMINANTS OF CHLAMYDIA TRACHOMATIS INFECTION AMONG SEXUALLY ACTIVE WOMEN IN TURIN - ITALY}

doi:10.1136/sextrans-2013-051184.0487

D De Maria, G De Intinis, M Peretto, V Buselli, M A Latino. Sant'Anna Hospital, Turin Italy

Background According to the WHO, Chlamydia trachomatis $(\mathrm{Ct})$ is the most common sexually transmitted bacterial agent worldwide. The infection is most common among young women less than 25 years old; the symptoms are often mild or inexistent.

The aim of this study was to assess the prevalence of Chlamydia trachomatis in women in Turin and to identify risk factors associated with this STI.

Methods This study was performed between January 2007 and December 2011, among sexually active women aged 14 to 65 years (mean 33 years) attending STI Centre of Sant'Anna Hospital in Turin, Italy. Every woman was submitted to a questionnaire aimed at collecting socio-demographic, behavioural and clinical information. Cervical specimens were tested with a real time qualitative PCR (Nanogen).

Chi square test and EpiInfo were used to identify associations between potential risk factors and $\mathrm{Ct}$ infection.

Results A total of 14071 women were included in the study. The overall prevalence of $\mathrm{Ct}$ was $2.8 \%$.

The prevalence rate in the symptomatic and asymptomatic patients was $3.5 \%$ versus $2.2 \%$; high prevalence rates were observed among women aged $14-24$ years $(9.9 \%$; $p<0.001)$, reporting more than one partner in the previous six months $(13.3 \%$; $<<0.001)$, among foreign women compared to Italian women (3.7\% vs $2.6 \%$; $\mathrm{p}<0.001)$. With regard to numbers of sexual partners lifetime, the prevalence rate for patients who had between 1 and 3 partners was $1.9 \%$ while in women with more than 3 partners was $4.1 \%$ $(p<0.001)$. In multivariate analysis, Ct was significantly associated with young age, more than 1 partner in the previous 6 months and use of barrier contraceptives.

Conclusion The value and risk factors highlighted can be compared with other studies. The high number of asymptomatic cases confirms the need to consider selective screening in order to reduce the spread of this STI and other secondary complications.

\section{P3.028 POSITIVITY RATE OF CHLAMYDIA TRACHOMATIS AND STATUS QUO OF OPPORTUNISTIC SCREENING IN GERMANY}

doi:10.1136/sextrans-2013-051184.0488

S Dudareva-Vizule, A Sailer, 0 Hamouda, V Bremer. Robert Koch Institute, Berlin, Germany

Background Chlamydia trachomatis (CT) infections are not reportable in Germany and limited data on CT-prevalence among women is available. In 2008 an opportunistic CT screening programme for women $<25$ years (OCS) was introduced. We assessed positivity rate of CT-infection and evaluated the OCS in order to develop recommendations to improve OCS.

Methods In September 2010 voluntary laboratory-based sentinel surveillance system was implemented and is collecting retrospective (from 2008) and prospective data on performed CT-tests together with results and information on age and test reason (TR). We calculated positivity rates (PR) by age and TR and compared them with $\mathrm{Chi}^{2}$ test. We described data by time and estimated the proportion of the target population reached by OCS.

Results As of 27.11.2012 data from 14 laboratories were available for analysis. These data cover approximately $25 \%$ of all CT-tests performed in Germany. Overall $90.6 \%(643,332)$ of all tests $(710,021)$ were among women. Among those $19.1 \%(122,650)$ 